\title{
Normality of meromorphic functions and their differential polynomials
}

\author{
Jia Xie ${ }^{\mathrm{a}}$, Yongyi Gu ${ }^{\mathrm{b}, *}$, Wenjun Yuan ${ }^{\mathrm{c}, *}$ \\ a School of Mathematics and Information Science, Guangzhou University, Guangzhou 510006 China \\ b Big data and Educational Statistics Application Laboratory, Guangdong University of Finance and \\ Economics, Guangzhou 510320 China \\ c Department of Basic Courses Teaching, Software Engineering Institute of Guangzhou, Guangzhou \\ 510990 China
}

*Corresponding authors, e-mail: gdguyongyi@163.com, wjyuan1957@126.com

Received 24 Feb 2021

Accepted 4 Jun 2021

ABSTRACT: In this paper, we study the normality of meromorphic families and prove the following theorem: Let $k$ be a positive integer, $P(z)$ be a non-constant polynomial satisfying $P(0)=0, h(\not \equiv 0)$ be a holomorphic function in a domain $D, H\left(f, f^{\prime}, \ldots, f^{(k)}\right)$ be a differential polynomial with $\left.\frac{\Gamma}{\gamma}\right|_{H}<k+1$, and $\mathscr{F}$ be a meromorphic family in $D$. If, for each $f \in \mathscr{F}, f \neq 0$ and $P\left(f^{(k)}\right)+H\left(f, f^{\prime}, \ldots, f^{(k)}\right) \neq h$ for $z \in D$, then $\mathscr{F}$ is a normal family in $D$.

KEYWORDS: meromorphic functions, differential polynomials, normality

MSC2010: 30D45

\section{INTRODUCTION AND MAIN RESULTS}

In this paper, we suppose the reader is acquainted with standard symbols and primary results on Nevanlinna theory $[1,2]$.

At first, we give some definitions about differential monomial and differential polynomial.

Definition 1 Let $f$ be a meromorphic function in domain $D$, and $n_{i}$ be positive integers for all $i \in$ $\{0,1, \ldots, k\}$. We say that $M\left(f, f^{\prime}, \ldots, f^{(k)}\right)$ is a differential monomial of $f$, if

$$
M\left(f, f^{\prime}, \ldots, f^{(k)}\right)=f^{n_{0}}\left(f^{\prime}\right)^{n_{1}} \cdots\left(f^{(k)}\right)^{n_{k}},
$$

where the degree of $M\left(f, f^{\prime}, \ldots, f^{(k)}\right)$ is $\gamma_{M}=n_{0}+$ $n_{1}+\cdots+n_{k}$, and the weight of $M\left(f, f^{\prime}, \ldots, f^{(k)}\right)$ is $\Gamma_{M}=n_{0}+2 n_{1}+\cdots+(k+1) n_{k}$.

Definition 2 Let $M_{1}\left(f, f^{\prime}, \ldots, f^{(k)}\right), M_{2}\left(f, \ldots, f^{(k)}\right)$, $\ldots, M_{n}\left(f, \ldots, f^{(k)}\right)$ be differential monomials of $f$, and $a_{1}(z), a_{2}(z), \ldots, a_{n}(z)$ analytic in $D$. Then $H\left(f, f^{\prime}, \ldots, f^{(k)}\right)$ is called a differential polynomial of $f$, if

$$
\begin{aligned}
& H\left(f, f^{\prime}, \ldots, f^{(k)}\right) \\
& \quad=a_{1}(z) M_{1}\left(f, \ldots, f^{(k)}\right)+\cdots+a_{n}(z) M_{n}\left(f, \ldots, f^{(k)}\right),
\end{aligned}
$$

where the degree of $H\left(f, f^{\prime}, \ldots, f^{(k)}\right)$ is $\gamma_{H}$ $=\max \left\{\gamma_{M_{1}}, \gamma_{M_{2}}, \ldots, \gamma_{M_{n}}\right\}$, and the weight of
$H\left(f, f^{\prime}, \ldots, f^{(k)}\right)$ is $\Gamma_{H}=\max \left\{\Gamma_{M_{1}}, \Gamma_{M_{2}}, \ldots, \Gamma_{M_{n}}\right\}$. If $\gamma_{M_{1}}=\gamma_{M_{2}}=\cdots=\gamma_{M_{n}}=m$, then $H\left(f, f^{\prime}, \ldots, f^{(k)}\right)$ is called the homogeneous differential polynomial of degree $m$. Set

$$
\left.\frac{\Gamma}{\gamma}\right|_{H}=\max \left\{\frac{\Gamma_{M_{1}}}{\gamma_{M_{1}}}, \frac{\Gamma_{M_{2}}}{\gamma_{M_{2}}}, \ldots, \frac{\Gamma_{M_{n}}}{\gamma_{M_{n}}}\right\} .
$$

In 1959, Hayman [3] proved the following result.

Theorem 1 Let $k$ be a positive integer and $f$ be a nonconstant meromorphic function in $\mathbb{C}$. Then $f$ or $f^{(k)}-1$ has at least a zero. Moreover, if $f$ is transcendental, then $f$ or $f^{(k)}-1$ has infinitely many zeros.

The normality corresponding to Theorem 1 was conjectured by Hayman [4] in 1967, and confirmed by $\mathrm{Gu}$ [5] in 1979 .

Theorem 2 Let $k$ be a positive integer and let $\mathscr{F}$ be a meromorphic family in a domain $D$. If, for each $f \in \mathscr{F}, f \neq 0$ and $f^{(k)} \neq 1$ for $z \in D$, then $\mathscr{F}$ is a normal family in $D$.

In 1986, Yang [6] extended Theorem 2 as follows.

Theorem 3 Let $k$ be a positive integer, $h(\not \equiv 0)$ be a holomorphic function in a domain $D$, and $\mathscr{F}$ be a 
meromorphic family in $D$. If for each $f \in \mathscr{F}, f \neq 0$ and $f^{(k)} \neq h$ for $z \in D$, then $\mathscr{F}$ is a normal family in $D$.

In 1993, Yang [6] replaced $f^{(k)}$ in Theorem 2 by a linear differential polynomial, and proved the following result.

Theorem 4 Let $k$ be a positive integer, $a_{1}(z), \ldots$, $a_{k}(z)$ be holomorphic functions in a domain $D$, and $\mathscr{F}$ be a meromorphic family in $D$. If for each $f \in \mathscr{F}$, $f \neq 0$ and $f^{(k)}(z)+a_{1}(z) f^{(k-1)}+\cdots+a_{k}(z) f(z) \neq 1$ for $z \in D$, then $\mathscr{F}$ is a normal family in $D$.

In 1991, Gu [7] considered the situation of homogeneous differential polynomial with constant coefficient, and proved the following result.

Theorem 5 Let $k, q \geqslant 3$ be two positive integers, $H\left(f, f^{\prime}, \ldots, f^{(k)}\right)=a_{1} M_{1}\left(f, f^{\prime}, \ldots, f^{(k)}\right)+\cdots+$ $a_{n} M_{n}\left(f, f^{\prime}, \ldots, f^{(k)}\right)$ be a homogeneous differential polynomial of degree $q$ with constant coefficient, and for each $i \in\{1, \ldots, n\}$, the degree of $f^{(k)}$ in $M_{i}\left(f, f^{\prime}, \ldots, f^{(k)}\right)$ be $\leqslant q-2$, and let $\mathscr{F}$ be a meromorphic family in a domain $D$. If for each $f \in \mathscr{F}$, $f \neq 0$ and $\left(f^{(k)}\right)^{q}+H\left(f, f^{\prime}, \ldots, f^{(k)}\right) \neq 1$ for $z \in D$, then $\mathscr{F}$ is a normal family in $D$.

In this paper, we improve Theorem 5 as follows.

Theorem 6 Let $k$ be a positive integer, $P(z)$ be a non-constant polynomial satisfying $P(0)=0$, $h(\not \equiv 0)$ be a holomorphic function in a domain $D$, $H\left(f, f^{\prime}, \ldots, f^{(k)}\right)$ be a differential polynomial with $\left.\frac{\Gamma}{\gamma}\right|_{H}<k+1$, and $\mathscr{F}$ be a meromorphic family in $D$. If for each $f \in \mathscr{F}, f \neq 0$ and $P\left(f^{(k)}\right)+$ $H\left(f, f^{\prime}, \ldots, f^{(k)}\right) \neq h$ for $z \in D$, then $\mathscr{F}$ is a normal family in $D$.

The following example shows that the condition $P(0)=0$ is necessary.

Example 1 Suppose that $\mathscr{F}=\left\{f_{n}=1 / n z\right\}, P(z)=$ $z^{2}+1$, and $D=\{z:|z|<1\}$. Then, for each $f_{n} \in \mathscr{F}$, $f_{n} \neq 0$ and $P\left(f_{n}^{(k)}\right)=(k !)^{2} / n^{2} z^{2 k+2}+1 \neq 1$ for $z \in D$, but $\mathscr{F}$ is not a normal family in $D$.

In 2000, Fang and Hong [8] proved the following result.

Theorem 7 Let $k, q \geqslant 2$ be two positive integers, $H\left(f, f^{\prime}, \ldots, f^{(k)}\right)$ be a differential polynomial with $\left.\frac{\Gamma}{\gamma}\right|_{H}<k+1$, and $\mathscr{F}$ be a meromorphic family in a domain $D$. If for each $f \in \mathscr{F}$, the multiplicity of zeros of $f$ are at least $k+1$ and $\left(f^{(k)}\right)^{q}+H\left(f, f^{\prime}, \ldots, f^{(k)}\right) \neq$ 1 for $z \in D$, then $\mathscr{F}$ is a normal family in $D$.
In this paper, we improve Theorem 7 slightly as follows.

Theorem 8 Let $k, q$ be two positive integers, $P(z)$ be a non-constant polynomial satisfying $P(z) \not \equiv-(1-$ $z)^{q}+1$ and $P(0)=0, H\left(f, f^{\prime}, \ldots, f^{(k)}\right)$ be a differential polynomial with $\left.\frac{\Gamma}{\gamma}\right|_{H}<k+1$, and $\mathscr{F}$ be a meromorphic family in a domain $D$. If for each $f \in \mathscr{F}$, the multiplicity of zeros of $f$ are at least $k+1$ and $P\left(f^{(k)}\right)+H\left(f, f^{\prime}, \ldots, f^{(k)}\right) \neq 1$ for $z \in D$, then $\mathscr{F}$ is a normal family in $D$.

The following example shows that the condition $P(z) \not \equiv-(1-z)^{q}+1$ is necessary.

Example 2 Suppose that $\mathscr{F}=\left\{f_{n}=\frac{1}{k !} \frac{z^{k+1}}{z+1 / n}\right\}$, $P(z)=-(1-z)^{q}+1$ with any positive integer $q$, and $D=\{z:|z|<1\}$. Then, for each $f_{n} \in \mathscr{F}$, the zeros of $f_{n}$ are multiplicity $\geqslant k+1, f_{n}^{(k)}=1-\frac{1}{(n z+1)^{k+1}} \neq 1$, and $P\left(f_{n}^{(k)}\right)=-\frac{1}{(n z+1)^{q k+q}}+1 \neq 1$ for $z \in D$, but $\mathscr{F}$ is not a normal family in $D$.

\section{LEMMAS}

In order to prove our results, we need some lemmas as follows.

Lemma 1 ([9]) Let $\alpha \in \mathbb{R}$ satisfy $-1<\alpha<\infty$ and $\mathscr{F}$ be a meromorphic family in a domain $D$. If $\mathscr{F}$ is not a normal family at $z_{0} \in D$, then there exist points $z_{n}(\in D) \rightarrow z_{0}$, functions $f_{n} \in \mathscr{F}$, and positive numbers $\rho_{n} \rightarrow 0^{+}$, such that $g_{n}(\xi)=\rho_{n}^{-\alpha} f\left(z_{n}+\rho_{n} \xi\right)$ converges locally spherically uniformly in $\mathbb{C}$ to a nonconstant meromorphic function $g(\xi)$, and moreover, $g$ is of order at most two.

Lemma 2 Let $f$ be a meromorphic function, $k$ be a positive integer, and $P(z)$ be a non-constant polynomial with $P(0)=0$. If $f \neq 0$ and $P\left(f^{(k)}\right) \neq 1$, then $f$ must be a constant.

Proof: Since $P(z)$ is a non-constant polynomial and $P(0)=0$, there exist a point $\omega(\neq 0) \in \mathbb{C}$ such that $P(\omega)-1=0$, and then we have $f^{(k)}(z) \neq \omega$ by $P\left(f^{(k)}\right) \neq 1$. It follows from Hayman's inequality and $f(z) \neq 0$ that $T(r, f) \leqslant S(r, f)$. That is $f(z) \equiv C$, where $C$ is a constant.

Lemma 3 ([10]) Let $k$ be a positive integer, let $q(z)$ and $p(z)$ are two coprime polynomials with $\operatorname{deg} q(z)<\operatorname{deg} p(z)$, and let $f(z)=a_{n} z^{n}+a_{n-1} z^{n-1}+$ $\cdots+a_{0}+\frac{q(z)}{p(z)}$, where $a_{0}, a_{1}, \ldots, a_{n}$ are constants with $a_{n} \neq 0$. If $f^{(k)}(z) \neq 1$, then we have

(i) $n=k$, and $k ! a_{k}=1$; 
(ii) $f(z)=\frac{1}{k !} z^{k}+\ldots+a_{0}+\frac{1}{(a z+b)^{m}}$;

(iii) If the zeros of $f(z)$ are of order at least $k+1$, then $m=1$ in (ii) and $f(z)=\frac{(c z+d)^{k+1}}{a z+b}$, where $c(\neq 0), d$ are constants.

Lemma 4 ([11]) Let $f$ be a transcendental meromorphic function in the complex plane, $k \geqslant 1$ be an integer, and $\varepsilon>0$. Then we have

$$
(1-\varepsilon) T(r, f) \leqslant \bar{N}\left(r, \frac{1}{f}\right)+N\left(r, \frac{1}{f^{(k)}-1}\right)+S(r, f) .
$$

Lemma 5 Let $k, q$ be two positive integers, let $P(z)$ be a non-constant polynomial with $P(0)=0$, and let $f(z)$ be a meromorphic function with finite order. If the zeros of $f(z)$ are of order $\geqslant k+1$ and $P\left(f^{(k)}\right) \neq 1$, then

(I) $f(z) \equiv C$, where $C$ is a constant, if $P(z) \not \equiv-(1-z)^{q}+1$;

(II) $f(z) \equiv C$ or $f(z) \equiv \frac{(c z+d)^{k+1}}{a z+b}$, if $P(z) \equiv-(1-z)^{q}+1$.

Proof: Since $P(z)$ is a non-constant polynomial and $P(0)=0$, there exists a point $\omega(\neq 0) \in \mathbb{C}$ such that $P(\omega)-1=0$. Without loss of generality we suppose that $\omega=1$. It is obvious that $f^{(k)}(z) \neq 1$ by $P\left(f^{(k)}(z)\right) \neq 1$.

We claim that $f(z)$ is a rational function. Presume that $f(z)$ is a transcendental meromorphic function of finite order. Then by Lemma 4 , setting $\varepsilon=1 / 2$ in (1) and taking into consideration of the zeros of $f(z)$ are multiplicity $\geqslant k+1 \geqslant 3$, we obtain that

$$
T(r, f) \leqslant 6 N\left(r, \frac{1}{f^{(k)}-1}\right)+S(r, f) .
$$

Clearly, (2) is a contradiction of $f^{(k)}(z) \neq 1$. That is $f(z)$ is a rational function. Now we consider two cases.

Case 1 Suppose that $f(z)$ is a rational function $q(z) / p(z)$, where $q(z)$ and $p(z)$ are coprime polynomial with $\operatorname{deg} p(z)>0$. Then by Lemma 3 we have $f(z)=q(z) / p(z)=(c z+d)^{k+1} /(a z+b)$. Hence $f^{(k)}(z)=1+(-1)^{k} k ! a^{k} /(a z+b)^{k+1}$, then $P(z) \equiv-(1-z)^{q}+1$ in this case.

In fact, if $P(z) \not \equiv-(1-z)^{q}+1$, then $P(z) \equiv$ $-(1-z)^{q}+1+\varphi(z)$, where $\varphi(z)$ is a polynomial with $\varphi(0)=0$ and $\varphi(z) \not \equiv(1-z)^{q}-(1-z)^{m}$, and $m$ is a nonnegative integer.

We claim that there exists a zero $\alpha(\neq 1)$ such that $P(\alpha)-1=-(1-\alpha)^{q}+\varphi(\alpha)=0$. Otherwise, $P(z)-1=0$ has only one zero $z=1$ that is $-(1-z)^{q}+\varphi(z)=A(1-z)^{m}$, where $A \neq 0$ is a constant and $m$ is a positive integer. Then we have
$\varphi(z)=A(1-z)^{m}+(1-z)^{q}$. By $\varphi(0)=A+1=0$ we have $A=-1$, so $\varphi(z)=-(1-z)^{m}+(1-z)^{q}$, a contradiction.

Hence there exists a point $\alpha(\neq 1)$ such that $P(\alpha)-1=0$. By $\alpha \neq 1$ we know that

$$
f^{(k)}(z)-\alpha=1+\frac{(-1)^{k} k ! a^{k}}{(a z+b)^{k+1}}-\alpha=0
$$

has solutions, which contradicts $P\left(f^{(k)}\right) \neq 1$.

Case 2 Suppose that $f(z)$ is a non-constant polynomial, then $\operatorname{deg} f \geqslant k+1$, hence $f^{(k)}(z)$ is a polynomial with $\operatorname{deg} f^{(k)} \geqslant 1$. Therefore $f^{(k)}=1$ has solutions, which contradicts $f^{(k)}(z) \neq 1$. Hence $f(z)$ is a constant.

\section{THE PROOF OF Theorem 6}

Proof: At first we explain that $\mathscr{F}$ is normal in the set $D^{\prime}=\{z \in D: h(z) \neq 0\}$. Presume that $\mathscr{F}$ is not normal at $z_{0} \in D^{\prime}$. Without loss of generality we may assume that $h\left(z_{0}\right)=1$. By Lemma 1 , there exist $f_{n} \in \mathscr{F}, z_{n} \rightarrow z_{0}$ and $\rho_{n} \rightarrow 0^{+}$such that $g_{n}(\xi)=$ $\rho_{n}^{-k} f_{n}\left(z_{n}+\rho_{n} \xi\right)$ converges locally spherically uniformly in $\mathbb{C}$ to a nonconstant meromorphic function $g(\xi)$. And by Hurwitz's theorem we know that $g(\xi) \neq 0$. As

$$
\begin{aligned}
P\left(g_{n}^{(k)}(\xi)\right) & -1=P\left(f_{n}^{(k)}\left(z_{n}+\rho_{n} \xi\right)\right)-1 \\
= & P\left(f_{n}^{(k)}\left(z_{n}+\rho_{n} \xi\right)\right) \\
& +H\left(f_{n}\left(z_{n}+\rho_{n} \xi\right), \ldots, f_{n}^{(k)}\left(z_{n}+\rho_{n} \xi\right)\right)-1 \\
& -H\left(f_{n}\left(z_{n}+\rho_{n} \xi\right), \ldots, f_{n}^{(k)}\left(z_{n}+\rho_{n} \xi\right)\right),
\end{aligned}
$$

and

$$
\begin{aligned}
& H\left(f_{n}\left(z_{n}+\rho_{n} \xi\right), \ldots, f_{n}^{(k)}\left(z_{n}+\rho_{n} \xi\right)\right) \\
& =\sum_{i=1}^{m} a_{i}\left(z_{n}+\rho_{n} \xi\right) M_{i}\left(f_{n}\left(z_{n}+\rho_{n} \xi\right), \ldots, f_{n}^{(k)}\left(z_{n}+\rho_{n} \xi\right)\right) \\
& =\sum_{i=1}^{m} a_{i}\left(z_{n}+\rho_{n} \xi\right) \rho_{n}^{(k+1) \gamma_{M_{i}}-\Gamma_{M_{i}}} M_{i}\left(g_{n}(\xi), \ldots, g_{n}^{(k)}(\xi)\right) .
\end{aligned}
$$

Considering $a_{i}(z)$ are analytical on $D$ for $i=$ $1,2, \ldots, m$, we deduce that

$$
\left|a_{i}\left(z_{n}+\rho_{n} \xi\right)\right| \leqslant M\left(\frac{1+r}{2}, a_{i}(z)\right)<\infty
$$

for sufficiently large $n$. Hence we come to the conclusion from $\left.\frac{\Gamma}{\gamma}\right|_{H}<k+1$ that

$$
\lim _{n \rightarrow \infty} \sum_{i=1}^{m} a_{i}\left(z_{n}+\rho_{n} \xi\right) \rho_{n}^{(k+1) \gamma_{M_{i}}-\Gamma_{M_{i}}} M_{i}\left(g_{n}(\xi), \ldots, g_{n}^{(k)}(\xi)\right)
$$


Thus we know that

$$
\begin{gathered}
P\left[g^{(k)}(\xi)\right]-1=\lim _{n \rightarrow \infty}\left\{P\left[g_{n}^{(k)}(\xi)\right]+\sum_{i=1}^{m} a_{i}\left(z_{n}+\rho_{n} \xi\right)\right. \\
\left.\times \rho_{n}^{(k+1) \gamma_{M_{i}}-\Gamma_{M_{i}}} M_{i}\left(g_{n}(\xi), \ldots, g_{n}^{(k)}(\xi)\right)\right\}-h\left(z_{0}\right) \\
=\lim _{n \rightarrow \infty}\left\{P\left[f_{n}^{(k)}\left(z_{n}+\rho_{n} \xi\right)\right]\right. \\
\left.+H\left(f_{n}\left(z_{n}+\rho_{n} \xi\right), \ldots, f_{n}^{(k)}\left(z_{n}+\rho_{n} \xi\right)\right)-h\left(z_{n}+\rho_{n} \xi\right)\right\} .
\end{gathered}
$$

Since $P\left(f^{(k)}(z)\right)+H\left(f(z), \ldots, f^{(k)}(z)\right)-h(z) \neq 0$, by Hurwitz's theorem we deduce that $P\left(g^{(k)}(\xi)\right) \equiv 1$ or $P\left(g^{(k)}(\xi)\right) \neq 1$. If $P\left(g^{(k)}(\xi)\right) \equiv 1$, then there exists a value $\omega \in \mathbb{C}$ such that $g^{(k)}(\xi) \equiv \omega$ and $P(\omega)=1$, which contradicts $g(\xi) \neq 0$. Therefore, $P\left(g^{(k)}(\xi)\right) \neq 1$. Then we conclude that $g(\xi)$ is a constant by Lemma 2 , a contradiction.

Now we prove that $\mathscr{F}$ is normal at $\{z: h(z)=$ $0\}$. Without loss of generality we may assume that $h(0)=0$, and we distinguish two cases.

Case $1 P(z)$ has at least two distinct zeros such as $a$ and $b$. Suppose that $\mathscr{F}$ is not normal at 0 . Then by Lemma 1 , there exist $z_{n} \rightarrow 0, f_{n} \in \mathscr{F}$, and $\rho_{n} \rightarrow 0^{+}$, such that $g_{n}(\xi)=\rho_{n}^{-k} f_{n}\left(z_{n}+\rho_{n} \xi\right)$ converges locally spherically uniformly to a nonconstant meromorphic function $g(\xi)$ in $\mathbb{C}$. Obviously $g(\xi) \neq 0$.

Similarly to the previous argument, we have

$$
\begin{aligned}
& P\left[g^{(k)}(\xi)\right]=\lim _{n \rightarrow \infty} P\left[g_{n}^{(k)}(\xi)\right] \\
& =\lim _{n \rightarrow \infty}\left\{P\left[f_{n}^{(k)}\left(z_{n}+\rho_{n} \xi\right)\right]\right. \\
& \left.+H\left(f_{n}\left(z_{n}+\rho_{n} \xi\right), \ldots, f_{n}^{(k)}\left(z_{n}+\rho_{n} \xi\right)\right)-h\left(z_{n}+\rho_{n} \xi\right)\right\} \text {. }
\end{aligned}
$$

Since $P\left(f^{(k)}(z)\right)+H\left(f(z), \ldots, f^{(k)}(z)\right)-h(z) \neq 0$ and $g(\xi) \neq 0$, by Hurwitz's theorem we deduce that $P\left(g^{(k)}(\xi)\right) \neq 0$. Hence $g^{(k)}(\xi) \neq a, b$. By $a \neq b$, then one of $a$ and $b$ is not 0 . We can assume that $a \neq 0$, that is $g(\xi) \neq 0$ and $g^{(k)}(\xi) \neq a(\neq 0)$. Then from Hayman's inequality, we have $g(\xi)$ is a constant, that is a contradiction.

Case $2 P(z)$ only has one zero. Hence $P(z)=$ $a z^{q}(a \neq 0, q \geqslant 1)$. By $h(0)=0$, then there exists a real constant $\delta>0$ such that $\bar{\Delta}(0, \delta)=\{z:|z| \leqslant$ $\delta\} \subset D$, and $h(z) \neq 0$ in $\Delta^{\prime}(0, \delta)=\{z: 0<|z|<$ $\delta\}$. From the previous discussion we have $\mathscr{F}$ is normal in $\Delta^{\prime}(0, \delta)$. For each $\left\{f_{n}\right\} \subset \mathscr{F}$, there exists a subsequence that we may also note as $\left\{f_{n}\right\}$, such that $f_{n}(z)$ converges locally spherically uniformly to $f(z)$ (meromorphic function or $\infty$ ) in $\Delta^{\prime}(0, \delta)$. Now we separate two subcases.
Case $2.1 f(z) \not \equiv 0$. If $f(z) \not \equiv \infty$, then from Hurwitz's theorem we have $f(z) \neq 0$ in $\Delta^{\prime}(0, \delta)$. Hence

$$
\min _{|z|=\delta / 2}|f(z)|=A>0,
$$

where $A$ is a constant. Then

$$
\min _{|z|=\delta / 2}\left|f_{n}(z)\right|>\frac{A}{2}>0
$$

for sufficiently large $n$. Since $f_{n}(z)$ are zero-free meromorphic functions in $\Delta^{\prime}(0, \delta)$, then $1 / f_{n}(z)$ are holomorphic in $\Delta^{\prime}(0, \delta)$. Therefore, $1 / f_{n}(z)$ are holomorphic in $\bar{\Delta}(0, \delta / 2)$, and

$$
\max _{|z|=\delta / 2}\left|\frac{1}{f_{n}(z)}\right|<\frac{2}{A} .
$$

By the Maximum Principle, we have

$$
\max _{|z| \leqslant \delta / 2}\left|\frac{1}{f_{n}(z)}\right|<\frac{2}{A},
$$

That is

$$
\min _{|z| \leqslant \delta / 2}\left|f_{n}(z)\right|>\frac{A}{2}>0 .
$$

Hence there exists a subsequence of $\left\{f_{n}\right\}$ converges locally spherically uniformly in $\Delta(0, \delta / 2)$. That is $\mathscr{F}$ is normal in $\Delta(0, \delta / 2)$.

If $f \equiv \infty$, then $\left\{f_{n}\right\}$ converges locally spherically uniformly to $\infty$ in $\Delta^{\prime}(0, \delta)$. That is $f_{n}(z)$ converges locally spherically uniformly to $\infty$ in $\{z$ : $|z|=\delta / 2\}$. For any $M>0$ and sufficiently large $n$, we have

$$
\min _{|z|=\delta / 2}\left|f_{n}(z)\right|>M>0 .
$$

Similarly to the previous argument, there exists a subsequence of $\left\{f_{n}\right\}$ converges locally spherically uniformly in $\Delta(0, \delta / 2)$. Hence $\mathscr{F}$ is normal in $\Delta(0, \delta / 2)$.

Case $2.2 f(z) \equiv 0$. Then $\left\{f_{n}\right\}$ converges locally spherically uniformly to 0 in $\Delta^{\prime}(0, \delta)$. Hence both

$$
\frac{a\left(f_{n}^{(k)}(z)\right)^{q}+H\left(f_{n}(z), \ldots, f_{n}^{(k)}(z)\right)}{h(z)}
$$

and

$$
\left(\frac{a\left(f_{n}^{(k)}(z)\right)^{q}+H\left(f_{n}(z), \ldots, f_{n}^{(k)}(z)\right)}{h(z)}\right)^{\prime}
$$

also converge locally spherically uniformly to 0 . By 
Argument Principle, we have

$$
\begin{aligned}
\mid N\left(\frac{\delta}{2}, 0,\right. & \left.\frac{a\left(f_{n}^{(k)}\right)^{q}+H\left(f_{n}, \ldots, f_{n}^{(k)}\right)}{h}-1\right) \\
& -N\left(\frac{\delta}{2}, 0, \frac{1}{\frac{a\left(f_{n}^{(k)}\right)^{q}+H\left(f_{n}, \ldots, f_{n}^{(k)}\right)}{h}-1}\right) \mid \\
& =\left|\frac{1}{2 \pi i} \int_{|z|=\frac{\delta}{2}} \frac{\left(\frac{a\left(f_{n}^{(k)}\right)^{q}+H\left(f_{n}, \ldots, f_{n}^{(k)}\right)}{h}\right)^{\prime}}{\frac{a\left(f_{n}^{(k)}\right)^{q}+H\left(f_{n}, \ldots, f_{n}^{(k)}\right)}{h}-1} \mathrm{~d} z\right|<1
\end{aligned}
$$

for sufficiently large $n$. Thus it can be seen that

$$
\begin{array}{r}
N\left(\frac{\delta}{2}, 0, f_{n}\right) \leqslant N\left(\frac{\delta}{2}, 0, \frac{a\left(f_{n}^{(k)}\right)^{q}+H\left(f_{n}, \ldots, f_{n}^{(k)}\right)}{h}-1\right) \\
=N\left(\frac{\delta}{2}, 0, \frac{1}{\frac{a\left(f_{n}^{(k)}\right)^{q}+H\left(f_{n}, \ldots, f_{n}^{(k)}\right)}{h}-1}\right)
\end{array}
$$

for sufficiently large $n$. By $a\left(f_{n}^{(k)}\right)^{q}+H\left(f_{n}, \ldots, f_{n}^{(k)}\right)$ $\neq h$, we have that $f_{n}$ are holomorphic in $\Delta(0, \delta / 2)$. That is $\left\{f_{n}\right\}$ converges locally spherically uniformly to 0 in $\Delta(0, \delta / 2)$. Hence $\mathscr{F}$ is normal at 0 . In conclusion, $\mathscr{F}$ is a normal family in $D$.

\section{THE PROOF OF Theorem 8}

Proof: For any $z_{0} \in D$, presume that $\mathscr{F}$ is not normal at $z_{0}$. Then by Lemma 1 , there exist $f_{n} \in \mathscr{F}$, $z_{n} \rightarrow z_{0}$ and $\rho_{n} \rightarrow 0^{+}$, such that $g_{n}(\xi)=\rho_{n}^{-k} f_{n}\left(z_{n}+\right.$ $\rho_{n} \xi$ ) converges locally spherically uniformly in $\mathbb{C}$ to a nonconstant meromorphic function $g(\xi)$, and moreover, $g$ is of order at most two. By Hurwitz's theorem we know that the zeros of $g$ are multiplicity $\geqslant k+1$.

If $P\left(g^{(k)}(\xi)\right) \neq 1$, then we conclude that $g(\xi)$ is a constant by Lemma 5 , a contradiction. Thus there exists $\xi_{0}$ such that $P\left(g^{(k)}\left(\xi_{0}\right)\right)=1$. Clearly, $g\left(\xi_{0}\right) \neq \infty$. Therefore there exists $\delta>0$ such that $g(\xi)$ is holomorphic function in $D_{2 \delta}=\{\xi$ : $\left.\left|\xi-\xi_{0}\right|<2 \delta\right\}$. Thus $g_{n}^{(i)}(\xi)(i=0,1, \ldots, k)$ are holomorphic in $D_{\delta}=\left\{\xi:\left|\xi-\xi_{0}\right|<\delta\right\}$ for large $n$ and $g_{n}^{(i)}(\xi)(i=0,1, \ldots, k)$ converges uniformly to $g^{(i)}(\xi)(i=0,1, \ldots, k)$ on $\bar{D}_{\delta}=\left\{\xi:\left|\xi-\xi_{0}\right| \leqslant \delta\right\}$. As

$$
\begin{aligned}
P\left(g_{n}^{(k)}(\xi)\right)-1 & =P\left(f_{n}^{(k)}\left(z_{n}+\rho_{n} \xi\right)\right)-1 \\
& =P\left(f_{n}^{(k)}\left(z_{n}+\rho_{n} \xi\right)\right) \\
+ & H\left(f_{n}\left(z_{n}+\rho_{n} \xi\right), \ldots, f_{n}^{(k)}\left(z_{n}+\rho_{n} \xi\right)\right)-1 \\
- & H\left(f_{n}\left(z_{n}+\rho_{n} \xi\right), \ldots, f_{n}^{(k)}\left(z_{n}+\rho_{n} \xi\right)\right),
\end{aligned}
$$

and

$$
\begin{aligned}
& H\left(f_{n}\left(z_{n}+\rho_{n} \xi\right), \ldots, f_{n}^{(k)}\left(z_{n}+\rho_{n} \xi\right)\right) \\
& =\sum_{i=1}^{m} a_{i}\left(z_{n}+\rho_{n} \xi\right) M_{i}\left(f_{n}\left(z_{n}+\rho_{n} \xi\right), \ldots, f_{n}^{(k)}\left(z_{n}+\rho_{n} \xi\right)\right) \\
& =\sum_{i=1}^{m} a_{i}\left(z_{n}+\rho_{n} \xi\right) \rho_{n}^{(k+1) \gamma_{M_{i}}-\Gamma_{M_{i}}} M_{i}\left(g_{n}(\xi), \ldots, g_{n}^{(k)}(\xi)\right),
\end{aligned}
$$

considering $a_{i}(z)(i=1,2, \ldots, m)$ are analytic on $D$, we have

$$
\left|a_{i}\left(z_{n}+\rho_{n} \xi\right)\right| \leqslant M\left(\frac{1+r}{2}, a_{i}(z)\right)<\infty,
$$

with sufficiently large $n$. For $\left.\frac{\Gamma}{\gamma}\right|_{H}<k+1$ we conclude that

$$
\sum_{i=1}^{m} a_{i}\left(z_{n}+\rho_{n} \xi\right) \rho_{n}^{(k+1) \gamma_{M_{i}}-\Gamma_{M_{i}}} M_{i}\left(g_{n}(\xi), \ldots, g_{n}^{(k)}(\xi)\right)
$$

converges uniformly to 0 on $D_{\delta / 2}=\left\{\xi:\left|\xi-\xi_{0}\right|<\right.$ $\delta / 2\}$. Thus we know that

$$
\begin{aligned}
P\left(g_{n}^{(k)}(\xi)\right) & +\sum_{i=1}^{m}\left[a_{i}\left(z_{n}+\rho_{n} \xi\right) \rho_{n}^{(k+1) \gamma_{M_{i}}-\Gamma_{M_{i}}}\right. \\
& \left.\times M_{i}\left(g_{n}(\xi), \ldots, g_{n}^{(k)}(\xi)\right)\right]-1 \\
= & P\left(f_{n}^{(k)}\left(z_{n}+\rho_{n} \xi\right)\right) \\
& +H\left(f_{n}\left(z_{n}+\rho_{n} \xi\right), \ldots, f_{n}^{(k)}\left(z_{n}+\rho_{n} \xi\right)\right)-1
\end{aligned}
$$

converges uniformly to $P\left(g^{(k)}(\xi)\right)-1$ on $D_{\delta / 2}=\{\xi$ : $\left.\left|\xi-\xi_{0}\right|<\delta / 2\right\}$

Since $P\left(f^{(k)}\right)+H\left(f, f^{\prime}, \ldots, f^{(k)}\right) \neq 1$, by Hurwitz's theorem we deduce that $P\left(g^{(k)}(\xi)\right) \equiv 1$ on $D_{\delta / 2}=\left\{\xi:\left|\xi-\xi_{0}\right|<\delta / 2\right\}$. Thus there exists a point $\omega \in \mathbb{C}$ such that $P(\omega)=1$ and $g^{(k)}(\xi) \equiv \omega$, which contradicts the zeros of $g$ are multiplicity $\geqslant k+1$.

Acknowledgements: This work was supported by the NSF of China (11901111), Joint PHD Program of Guangzhou University and Curtin University, Visiting Scholar Program of Chern Institute of Mathematics at Nankai University when the authors worked as visiting scholars. The authors would like to express their hearty thanks to Chern Institute of Mathematics for very comfortable research environment.

\section{REFERENCES}

1. Hayman WK (1964) Meromorphic Functions, Clarendon Press, Oxford.

2. Laine I (2011) Nevanlinna Theory and Complex Differential Equations, Walter de Gruyter. 
3. Hayman WK (1959) Picard values of meromorphic functions and their derivatives. Ann Math 70, 9-42.

4. Hayman WK (1967) Research Problems in Function Theory, Athlone Press, London.

5. Gu YX (1979) A criterion for normality of families of meromorphic functions. Sci Sinica S1, 267-274.

6. Yang L (1993) Value Distribution Theory, Science Press, Beijing.

7. Gu YX (1991) Normal Families of Meromorphic Functions, Sichuan Education Press, Sichuan. [in Chinese]
8. Fang ML, Hong W (2000) Some results on normal family of meromorphic functions. Bull Malays Math Sci Soc 23, 143-151.

9. Pang XC, Zalcman L (2000) Normal families and shared values. Bull London Math Soc 32, 325-331.

10. Wang YF, Fang ML (1998) Picard values and normal families of meromorphic functions with multiple zeros. Acta Math Sinica 14, 17-26.

11. Fang ML, Wang YF (2013) A note on the conjectures of Hayman, Mues and Gol'dberg. Comput Methods Funct Theory, 13, 533-543. 